$$
\begin{aligned}
& G_{1 l}^{\mathrm{kr}} \sim \frac{-\sqrt{2 \pi}}{8(l+1) !} \frac{e^{i(\pi / 6)}}{6^{2 / 3}(k a)^{1 / 3}} \frac{1}{(k a \sin \vartheta)^{1 / 2} k r r^{\prime}\left(1-\frac{a^{2}}{r^{2}}\right)^{1 / 4}\left(1-\frac{a^{2}}{r^{\prime 2}}\right)^{1 / 4}} \\
& \cdot \exp \left\{i k\left[\left(r^{2}-a^{2}\right)^{1 / 2}+\left(r^{\prime 2}-a^{2}\right)^{1 / 2}+2(l+1) a\left(\frac{k_{\mathrm{i}}^{2}}{k^{2}}-1\right)^{1 / 2}\right]\right\} \cdot\left\{\frac{\delta_{\mathrm{a}}{ }^{2}}{8 \mathrm{i}^{2}} \frac{n^{2}}{n^{2}-1}\right\}\left\{\frac{4 \mathrm{Ba}^{2} n^{2}}{8 \mathrm{~B}^{2}\left(n^{2}-1\right)}\right\}^{\frac{l+1}{2}} \\
& \cdot \sum_{\nu_{\mathrm{P}}}\left\{\frac{\partial l+1}{\partial v^{l+1}} \frac{\exp \left\{-i v\left(\operatorname{arcos} \frac{a}{r}+\operatorname{arcos} \frac{a}{r^{\prime}}+2(l+1) \operatorname{arcos} \frac{k}{k_{\mathrm{i}}}\right)\right\}}{[A(q)]^{2} \cos v \pi}\right.
\end{aligned}
$$

$$
\left.\cdot\left[\exp \left\{i v(\vartheta-\pi)+i\left(v-\frac{1}{2}\right) 2 m_{1} \pi+i \frac{3 \pi}{4}\right\}+\exp \left\{i v(\pi-\vartheta)+i\left(v-\frac{1}{2}\right) 2 m_{2} \pi-i \frac{3 \pi}{4}\right\}\right]\right\}_{\nu_{\nu}=\nu_{\mathrm{P}}}\left(n=k_{\mathrm{i}} / k\right) .
$$

Auch hier liefert $l=0$ Beiträge des Linsenterms.

Der Verfasser ist Herrn Professor Dr. W WLter $F_{\text {RANZ }}$ für die Anregung und Förderung der vorliegenden Untersuchung zu aufrichtigem Dank verpflichtet.

\title{
Linsengleidhung und Bildkurve des Zylinder-Kondensators bei schiefem Ein- und Austritt der lonenbündel an den Feldgrenzen
}

\author{
Von H. Wende * \\ Aus dem Max-Planck-Institut für Chemie, Mainz \\ (Z. Naturforschg. 12 a, 967-970 [1957] ; eingegangen am 23. September 1957)
}

\begin{abstract}
Die ionenoptische Linsengleichung des Zylinderkondensators wird mittels der Theorie zweiter Näherung auf den Fall schiefen Ein- und Austritts an den Feldgrenzen verallgemeinert. Es werden Angaben über die Lage der Bildkurve und die Eigenschaften eines „Energie-Spektrographen“ gemacht.
\end{abstract}

Seit den grundlegenden Arbeiten von Herzog ${ }^{1}$ über elektrische Sektorfelder weiß man, daß diese sich gegen Ionen- bzw. Elektronenstrahlen ähnlich wie Linsen (und Prismen) gegen Lichtstrahlen verhalten und einer Linsengleichung

$$
\begin{gathered}
\left(l_{\mathrm{e}}^{\prime}-g_{\mathrm{e} 0}\right)\left(l_{\mathrm{e}}^{\prime \prime}-g_{\mathrm{e} 0}\right)=f_{\mathrm{e} 0}{ }^{2}, \\
g_{\mathrm{e} 0}=r_{\mathrm{e}} \frac{\sqrt{2}}{2} \cot \sqrt{2} \Phi_{\mathrm{e}}, \\
f_{\mathrm{e} 0}=r_{\mathrm{e}} \frac{\sqrt{2}}{2} \frac{1}{\sin \sqrt{2} \Phi_{e}}
\end{gathered}
$$

genügen (s. Abb. 1). Da diese Rechnungen für den Zylinderkondensator in erster Näherung ausgeführt wurden, gilt die Gleichung nur in der Nähe des Mittelstrahls bei nahezu senkrechtem Ein- und Austritt an den Feldgrenzen. Nach Gl. (1) existiert eine Bildebene, welche die Mittelbahn im Abstand $l_{\mathrm{e}}{ }^{\prime \prime}$ von der hinteren Feldgrenze schneidet. Über den Winkel, den diese Bildebene mit der Mittelbahn bildet, kann die Theorie erster Näherung ebenso-

* Die vorliegende Arbeit enthält wesentliche Bestandteile der Dissertation von H. WeNDE (D 77). wenig eine Aussage machen wie über das ionenoptische Verhalten bei schiefem Ein- und Austritt. Beim praktischen Arbeiten (z. B. dem Einjustieren

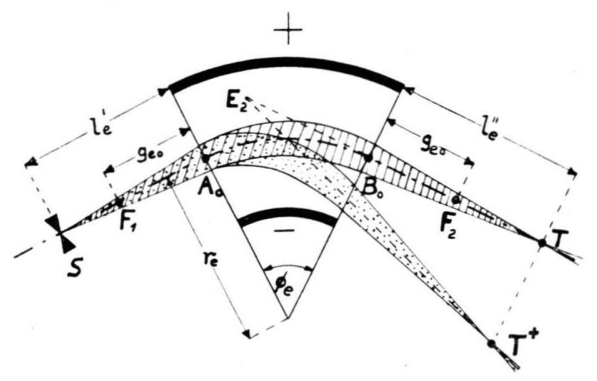

Abb. 1. Strahlengang im Zylinderkondensator für ein Bündel von Ionen zweier verschiedener Energien.

eines Massenspektrographen) hat man es sehr oft mit solchen schräg einfallenden Bündeln zu tun, so daß die Kenntnis ihrer ionenoptischen Eigenschaften sehr erwünscht ist. Seit einigen Jahren sind nun

1 R. Herzog, Z. Phys. 89, 447 [1934]. 
durch die Arbeiten von Hintenberger ${ }^{2}$ und VoorHIEs $^{3}$ die Bahngleichungen im Zylinderkondensator auch in zweiter Näherung bekannt. Mit ihrer Hilfe soll im folgenden die Linsengleichung für schiefen Einschuß verallgemeinert und die Lage der Bildkurve berechnet werden.

Von dem als punktförmig angenommenen Spalt S mögen Ionen der Masse $M$ mit der Geschwindigkeit $v=v_{0}(1+\beta)$ ausgehen, wobei $M v_{0}^{2} / r_{\mathrm{e}}=e E$ ist ( $e=$ Elementarladung, $E=$ Elektr. Feldstärke auf der Mittelbahn). Für die Austrittsordinate $y_{0}$ und den Austrittswinkel $\varepsilon^{\prime \prime}$ einer Ionenbahn am Zylinder- kondensator erhält man ${ }^{2}$ mit den Abkürzungen $\lambda \equiv l_{\mathrm{e}}^{\prime} / r_{\mathrm{e}}$ und $\sigma \equiv s / r_{\mathrm{e}}$ (s. Abb. 2)

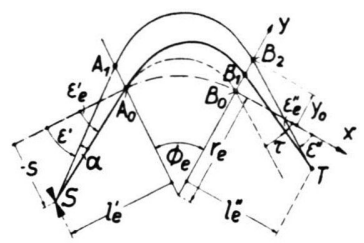

Abb. 2. Ionenstrahlen im Zylinderkondensator (zur Aufstellung der Linsengleichung).

$$
\begin{aligned}
\frac{y_{0}}{r_{\mathrm{e}}}= & \cos \sqrt{2} \Phi_{\mathrm{e}}+\varepsilon^{\prime}\left(\frac{\sqrt{2}}{2} \sin \sqrt{2} \Phi_{\mathrm{e}}+\lambda \cos \sqrt{2} \Phi_{\mathrm{e}}\right)+\beta\left(1-\cos \sqrt{2} \Phi_{\mathrm{e}}\right)+\sigma^{2}\left(-\frac{3}{4}+\frac{1}{6} \cos \sqrt{2} \Phi_{\mathrm{e}}\right. \\
& \left.+\frac{7}{12} \cos 2 \sqrt{2} \Phi_{\mathrm{e}}\right)+\sigma \varepsilon^{\prime}\left(-\frac{3}{2} \lambda-\frac{2 \sqrt{2}}{3} \sin \sqrt{2} \Phi_{\mathrm{e}}+\frac{1}{3} \lambda \cos \sqrt{2} \Phi_{\mathrm{e}}+\frac{7 \sqrt{2}}{12} \sin 2 \sqrt{2} \Phi_{\mathrm{e}}+\frac{7}{6} \lambda \cos 2 \sqrt{2} \Phi_{\mathrm{e}}\right) \\
& +\sigma \beta\left(\frac{3}{2}-\frac{1}{3} \cos \sqrt{2} \Phi_{\mathrm{e}}-\frac{7}{6} \cos 2 \sqrt{2} \Phi_{\mathrm{e}}\right)+\varepsilon^{\prime 2}\left(-\frac{3}{8}-\frac{3}{4} \lambda^{2}-\frac{2 \sqrt{2}}{3} \sin \sqrt{2} \Phi_{\mathrm{e}}+\left[\frac{2}{3}+\frac{1}{6} \lambda^{2}\right] \cos \sqrt{2} \Phi_{\mathrm{e}}\right. \\
& \left.+\frac{7 \sqrt{2}}{12} \lambda \sin 2 \sqrt{2} \Phi_{\mathrm{e}}-\left[\frac{7}{24}-\frac{7}{12} \lambda^{2}\right] \cos 2 \sqrt{2} \Phi_{\mathrm{e}}\right)+\varepsilon^{\prime} \beta\left(\frac{3}{2} \lambda+\frac{7 \sqrt{2}}{6} \sin \sqrt{2} \Phi_{\mathrm{e}}-\frac{1}{3} \lambda \cos \sqrt{2} \Phi_{\mathrm{e}}\right. \\
- & \left.\frac{7 \sqrt{2}}{12} \sin 2 \sqrt{2} \Phi_{\mathrm{e}}-\frac{7}{6} \lambda \cos 2 \sqrt{2} \Phi_{\mathrm{e}}\right)+\beta^{2}\left(\frac{1}{4}-\frac{5}{6} \cos \sqrt{2} \Phi_{\mathrm{e}}+\frac{7}{12} \cos 2 \sqrt{2} \Phi_{\mathrm{e}}\right), \\
-\varepsilon^{\prime \prime}= & \sigma\left(-\sqrt{2} \sin \sqrt{2} \Phi_{\mathrm{e}}\right)+\varepsilon^{\prime}\left(-\sqrt{2} \lambda \sin \sqrt{2} \Phi_{\mathrm{e}}+\cos \sqrt{2} \Phi_{\mathrm{e}}\right)+\beta \sqrt{2} \sin \sqrt{2} \Phi_{\mathrm{e}}+\sigma^{2}\left(-\frac{\sqrt{2}}{6} \sin \sqrt{2} \Phi_{\mathrm{e}}\right. \\
& \left.-\frac{2 \sqrt{2}}{3} \sin 2 \sqrt{2} \Phi_{\mathrm{e}}\right)+\sigma \varepsilon^{\prime}\left(-\frac{\sqrt{2}}{3} \lambda \sin \sqrt{2} \Phi_{\mathrm{e}}-\frac{4}{3} \cos \sqrt{2} \Phi_{\mathrm{e}}-\frac{4 \sqrt{2}}{3} \lambda \sin 2 \sqrt{2} \Phi_{\mathrm{e}}+\frac{4}{3} \cos 2 \sqrt{2} \Phi_{\mathrm{e}}\right) \\
& +\sigma \beta\left(\frac{4 \sqrt{2}}{3} \sin \sqrt{2} \Phi_{\mathrm{e}}+\frac{4 \sqrt{2}}{3} \sin 2 \sqrt{2} \Phi_{\mathrm{e}}\right)+\varepsilon^{\prime} 2\left(-\left[\frac{2 \sqrt{2}}{3}+\frac{\sqrt{2}}{6} \lambda^{2}\right] \sin \sqrt{2} \Phi_{\mathrm{e}}-\frac{4}{3} \lambda \cos \sqrt{2} \Phi_{\mathrm{e}}\right. \\
& \left.+\left[\frac{\sqrt{2}}{3}-\frac{2 \sqrt{2}}{3} \lambda^{2}\right] \sin 2 \sqrt{2} \Phi_{\mathrm{e}}+\frac{4}{3} \lambda \cos 2 \sqrt{2} \Phi_{\mathrm{e}}\right)+\varepsilon^{\prime} \beta\left(\frac{4 \sqrt{2}}{3} \lambda \sin \sqrt{2} \Phi_{\mathrm{e}}+\frac{4}{3} \cos \sqrt{2} \Phi_{\mathrm{e}}\right. \\
& \left.+\frac{4 \sqrt{2}}{3} \lambda \sin 2 \sqrt{2} \Phi_{\mathrm{e}}-\frac{4}{3} \cos 2 \sqrt{2} \Phi_{\mathrm{e}}\right)+\beta^{2}\left(-\frac{\sqrt{2}}{6} \sin \sqrt{2} \Phi_{\mathrm{e}}-\frac{2 \sqrt{2}}{3} \sin 2 \sqrt{2} \Phi_{\mathrm{e}}\right) .
\end{aligned}
$$

Wir betrachten nun ein Ionenbündel, dessen Leitstrahl am Schnittpunkt $A_{0}$ der Mittelbahn mit der Feldgrenze unter dem Winkel $\varepsilon_{\mathrm{e}}{ }^{\prime}$ in den Zylinderkondensator eintritt. Ein Teilstrahl dieses Bündels bilde mit dem Leitstrahl den Winkel $\alpha$. Der Öffnungswinkel des Bündels sei so klein, daß die Glieder mit $\alpha^{2}$ gegen die übrigen vernachlässigbar sind. Die Ionenenergie - und damit $\beta$ - hängt von $\varepsilon_{\mathrm{e}}^{\prime}$ und $\varepsilon_{\mathrm{e}}{ }^{\prime \prime}$ ab. Mit $\tau \equiv \varepsilon_{\mathrm{e}}^{\prime}-\varepsilon_{\mathrm{e}}^{\prime \prime}$ erhält man aus Gln. (2) und (3) durch Elimination von $y_{0}$

$$
\beta=\tau \frac{\sqrt{2}}{2} \frac{1}{\sin \sqrt{2} \Phi_{\mathrm{e}}}-\varepsilon_{\mathrm{e}}{ }^{\prime} \frac{\sqrt{2}}{2} \cot \frac{\sqrt{2} \Phi_{\mathrm{e}}}{2}+\text { Glieder 2. Ordnung. }
$$

Aus Abb. 2 liest man ab

$$
\varepsilon^{\prime}=\varepsilon_{\mathrm{e}}^{\prime}+\alpha, \quad(4 \mathrm{~b}) \quad s=-\varepsilon_{\mathrm{e}}^{\prime} l_{\mathrm{e}}^{\prime} \quad \text { bzw. } \quad \sigma=-\varepsilon_{\mathrm{e}}^{\prime} \lambda .
$$

Gln. (4a), (4b) und (4c) in Gl. (2) und (3) eingesetzt ergeben (bei Vernachlässigung der Glieder mit $\alpha^{2}$ )

$$
y_{0} / r_{\mathrm{e}}=F_{0}+\alpha F_{1} \quad(5 \mathrm{a}) \quad \text { und } \quad-\varepsilon^{\prime \prime}=G_{0}+\alpha G_{1} \text {, }
$$

2 Persönliche Mitteilung von Herrn Prof. H. Hintenberger im Juli 1954 .
3 H. G. Voorhies, Rev. Sci. Instrum. 26, 716 [1955] ; 27, 58 [1956]. 
wobei

$$
\begin{aligned}
F_{1}=\frac{\sqrt{2}}{2} \sin \sqrt{2} \Phi_{\mathrm{e}}+\lambda \cos \sqrt{2} \Phi_{\mathrm{e}}-\varepsilon_{\mathrm{e}}^{\prime}( & \left.\frac{4}{3}\left[1-\cos \sqrt{2} \Phi_{\mathrm{e}}\right]+2 \sqrt{2} \lambda \sin \sqrt{2} \Phi_{\mathrm{e}}\right) \\
& +\tau\left(\frac{7}{6}\left[1-\cos \sqrt{2} \Phi_{\mathrm{e}}\right]+\frac{\sqrt{2}}{6} \lambda\left[\operatorname{tg} \frac{\sqrt{2} \Phi_{\mathrm{e}}}{2}+7 \sin \sqrt{2} \Phi_{\mathrm{e}}\right]\right)
\end{aligned}
$$

und

$$
\begin{aligned}
G_{1}=-\sqrt{2} \lambda \sin \sqrt{2} \Phi_{\mathrm{e}}+\cos \sqrt{2} \Phi_{\mathrm{e}}-\varepsilon_{\mathrm{e}}{ }^{\prime}\left(2 \sqrt{2} \sin \sqrt{2} \Phi_{\mathrm{e}}+\frac{8}{3} \lambda\left[1+2 \cos \sqrt{2} \Phi_{\mathrm{e}}\right]\right) \\
+\tau\left(\frac{2 \sqrt{2}}{3}\left[2 \sin \sqrt{2} \Phi_{\mathrm{e}}-\operatorname{tg} \frac{\sqrt{2} \Phi_{\mathrm{e}}}{2}\right]+\frac{4}{3} \lambda\left[1+2 \cos \sqrt{2} \Phi_{\mathrm{e}}\right]\right) .
\end{aligned}
$$

Die Angabe von $F_{0}$ und $G_{0}$ erübrigt sich, da diese Glieder bei der weiteren Rechnung herausfallen.

Somit lautet die Bahngleichung des aus dem Zylinderkondensator austretenden Ionenstrahls in zweiter Näherung

$$
y=y_{0}-\varepsilon^{\prime \prime} x=\left(r_{\mathrm{e}} F_{0}+x G_{0}\right)+\alpha\left(r_{\mathrm{e}} F_{1}+x G_{1}\right) .
$$

Im Bildpunkt $\mathrm{T}$ muß $y$ unabhängig von $\alpha$ sein. Also (da dann $x=l_{\mathrm{e}}{ }^{\prime \prime}$ )

$$
r_{\mathrm{e}} F_{1}+l_{\mathrm{e}}^{\prime \prime} G_{1}=0 \text {. }
$$

Dies ist die gesuchte Linsengleichung. In der üblichen Schreibweise lautet sie:

$$
\left(l_{\mathrm{e}}{ }^{\prime}-g_{\mathrm{e}}{ }^{\prime}\right)\left(l_{\mathrm{e}}{ }^{\prime \prime}-g_{\mathrm{e}}{ }^{\prime \prime}\right)=f_{\mathrm{e}}{ }^{2}
$$

mit

$$
\begin{aligned}
g_{\mathrm{e}}{ }^{\prime}=g_{\mathrm{e} 0}\left(1+\varepsilon_{\mathrm{e}}{ }^{\prime} a_{1}+\tau a_{2}\right) ; & a_{1}=-2 \sqrt{2} \operatorname{tg} \sqrt{2} \Phi_{\mathrm{e}}-\frac{4 \sqrt{2}}{3} \frac{1+2 \cos \sqrt{2} \Phi_{\mathrm{e}}}{\sin \sqrt{2} \Phi_{\mathrm{e}}}=b_{1}, \\
g_{\mathrm{e}}{ }^{\prime \prime}=g_{\mathrm{e} 0}\left(1+\varepsilon_{\mathrm{e}}{ }^{\prime} b_{1}+\tau b_{2}\right) ; & a_{2}=\frac{4 \sqrt{2}}{3} \frac{1+2 \cos \sqrt{2} \Phi_{\mathrm{e}}}{\sin \sqrt{2} \Phi_{\mathrm{e}}}=-c_{1}, \\
f_{\mathrm{e}}=f_{\mathrm{e} 0}\left(1+\varepsilon_{\mathrm{e}}{ }^{\prime} c_{1}+\tau c_{2}\right) ; & b_{2}=\frac{\sqrt{2}}{6}\left(\frac{16}{\sin 2 \sqrt{2} \Phi_{\mathrm{e}}}+\frac{3}{\sin \sqrt{2} \Phi_{\mathrm{e}}}+\cot \sqrt{2} \Phi_{\mathrm{e}}\right), \quad c_{2}=\frac{\sqrt{2} \frac{11+13 \cos \sqrt{2} \Phi_{\mathrm{e}}}{12}}{\sin \sqrt{2} \Phi_{\mathrm{e}}}
\end{aligned}
$$

Für $\varepsilon_{\mathrm{e}}{ }^{\prime}=\tau=0$ geht sie in die aus der Theorie erster Näherung bekannte Gl. (1) über.

Durch eine elementare geometrische Überlegung (s. Abb. 3) findet man hieraus die Lage der Bildkurve. Sie bildet mit dem symmetrisch ein- und austretenden Strahl $(\tau=0)$ den Winkel $\psi$. Man erhält nach kurzer Rechnung

$$
\operatorname{tg} \psi=\frac{\gamma+\gamma^{2}}{a+b \gamma+c \gamma^{2}}, \quad \text { wobei } \quad \gamma \equiv \frac{l_{\mathrm{e}^{\prime}-g_{\mathrm{e} 0}}}{f_{\mathrm{e} 0}},
$$

$$
a=a_{2} \cos \sqrt{2} \Phi_{\mathrm{e}}, \quad b=2 c_{2}, \quad c=b_{2} \cos \sqrt{2} \Phi_{\mathrm{e}} .
$$

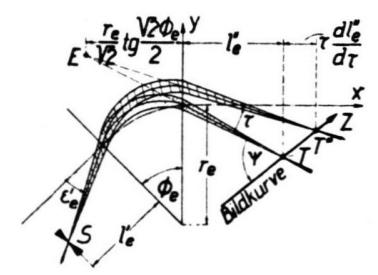

Abb. 3. Ionenbündel im Zylinderkondensator (zur Berechnung der Lage der Bildkurve). Der Abstand des „Emissionszentrums" $E$ von der Feldgrenze folgt aus der Theorie erster Näherung zu $f_{\mathrm{e} 0}-g_{\mathrm{e} 0}$.
In Abb. 4 ist $\psi$ als Funktion des Ablenkwinkels $\Phi_{\mathrm{e}}$ und der Spaltstellung $\gamma$ dargestellt.

Bringt man an den Ort der Bildkurve eine Photoplatte, dann kann man das Energiespektrum aufnehmen. Die Fokussierung verhält sich ähnlich wie die Abbildung in Massenspektrographen mit Doppelfokussierung in einem Punkt: Das Spektrum wird nur in der Umgebung eines Punktes (d.h. in der Nähe einer bestimmten Energie) scharf abgebildet. Wie bei den Spektrographen der Lichtoptik wird die Leistungsfähigkeit eines solchen „Energie-Spektrographen " durch Auflösung und Dispersion bestimmt. Das Energie-Auflösungsvermögen $A_{\mathrm{E}}$ folgt aus der Theorie erster Näherung zu

$$
A_{\mathrm{E}}=\frac{r_{\mathrm{e}}}{2 b_{\mathrm{s}}}\left(1+\frac{l_{\mathrm{e}^{\prime}}-f_{\mathrm{e}}}{f_{\mathrm{e}}}\right),
$$

wo $b_{\mathrm{s}}$ die Breite des Spaltes ( $\mathrm{S}$ in Abb. 3 ) bedeutet. Die Dispersion liest man aus Abb. 3 unter Benutzung von Gl. (4 a) ab: Enthält das in den Zylinderkondensator einfallende Bündel Ionen bzw. Elektronen der beiden Energien $E$ und $E+\Delta E$, so erscheinen ihre Bilder $\mathrm{T}$ und $\mathrm{T}^{*}$ auf einer in der Bild- 


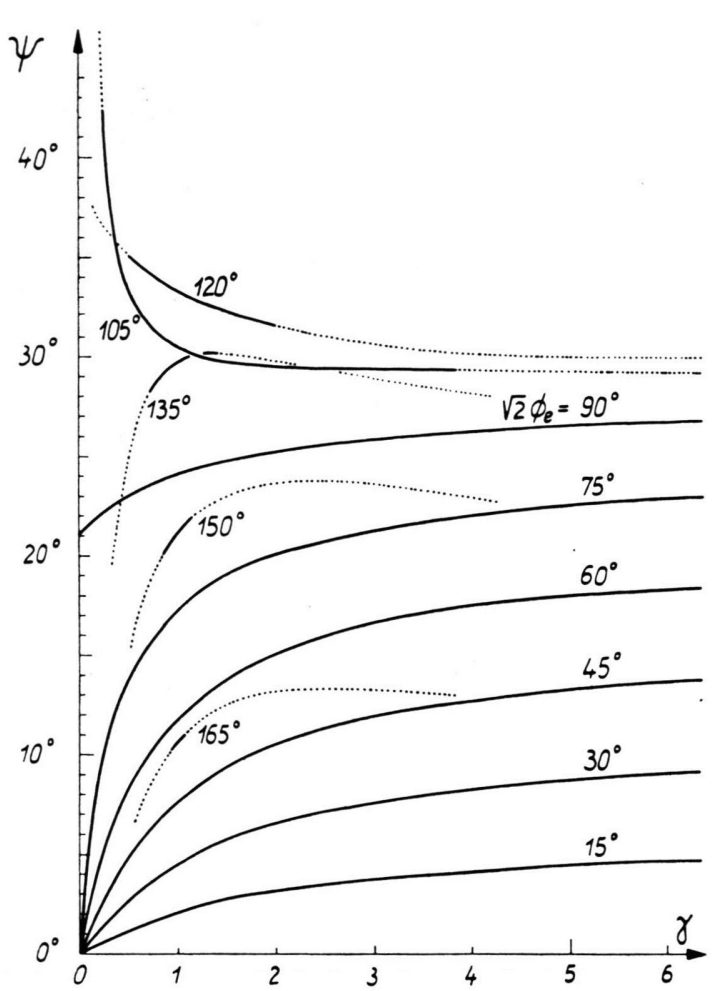

Abb. 4. Winkel $\psi$ zwischen Bildkurve und Ionenstrahl. Die ausgezogenen Kurvenstücke entsprechen Versuchsanordnungen, die sich durch passende Spaltstellung realisieren lassen. In den punktierten Kurventeilen ist entweder $l_{\mathrm{e}}^{\prime}<0$ (d. h. das in den Zylinderkondensator eintretende Bündel ist konvergent) oder $l_{\mathrm{e}}{ }^{\prime \prime}<0$ (d.h. die aus dem Zylinderkondensator austretenden Bündel sind divergent).

ebene angebrachten Photoplatte um

$$
\Delta z=D_{\mathrm{E}} \frac{\Delta E}{E}
$$

gegeneinander versetzt. Dabei ist

$$
D_{\mathrm{E}}=\frac{1+\frac{\sqrt{2}}{2}-\cos \sqrt{2} \Phi_{\mathrm{e}}+\frac{1}{\gamma}}{2 \sin \psi} r_{\mathrm{e}}
$$

der „Energie-Dispersionskoeffizient“. $\psi$ ist nach Gl. (7) zu berechnen bzw. aus Abb. 4 zu entnehmen. Als Zahlenbeispiel diene der in unserem MattauCHHerzog-Massenspektrographen verwendete Zylinderkondensator mit $r_{\mathrm{e}}=28 \mathrm{~cm}$ und $\sqrt{2} \Phi_{\mathrm{e}}=\pi / 4$. Für $\gamma=1$ (d. h. möglichst kleinen Abstand zwischen Spalt $\mathrm{S}$ und Bild T: $l_{\mathrm{e}}{ }^{\prime}=l_{\mathrm{e}}{ }^{\prime \prime}=478 \mathrm{~mm}$ ) ist nach Gl. (7) $\psi=7^{\circ} 4 \mathrm{l}^{\prime}$ und mithin wegen Gl. (9) $D_{\mathrm{E}}=2094 \mathrm{~mm}$. Bei einer Spaltweite $b_{\mathrm{s}}=2,8 \mu$ beträgt das Auflösungsvermögen $A_{\mathrm{E}}=100000$. Bei $100 \mathrm{kV}$ werden demnach Ionen mit einem Energieunterschied von $1 \mathrm{eV}$ gerade noch getrennt. In diesem Fall ist Linienbreite $=$ Linienabstand $=21 \mu$.

Wie man aus der Linsengleichung (6) ablesen kann, genügt schon eine geringfügige Änderung des nur schwer genau zu messenden Einschußwinkels $\varepsilon_{\mathrm{e}}{ }^{\prime}$, um $g_{\mathrm{e}}$ und die Brennweite $f_{\mathrm{e}}$ stark zu ändern. Es scheint, daß sich auf diese Wèise (durch leicht schiefen Einschuß) manche Diskrepanz (bzw. „Übereinstimmung“) zwischen theoretischer und beobachteter Brennweite erklären läßt. Jedenfalls sollte bei allen Brennweiten-Messungen $\varepsilon_{\mathrm{e}}{ }^{\prime}$ mit äußerster Sorgfalt bestimmt werden, um grobe Fehler zu vermeiden.

Am Schluß möchte ich Herrn Prof. Dr. H. HintenberGER für die freundliche Mitteilung der Bahngleichungen im Zylinderkondensator und meinem verehrten Lehrer, Herrn Prof. Dr. J. Mattauch, für die Förderung dieser Arbeit herzlich danken. 